\section{Identity of CAF revealed}

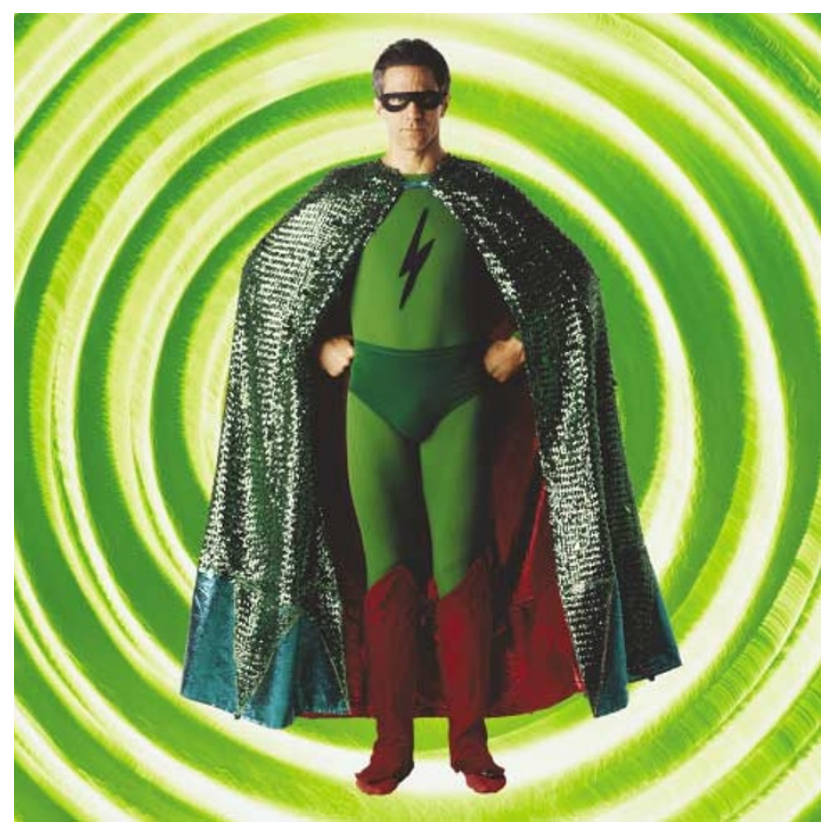

Until recently, it was a mystery why some individuals infected with HIV-1 do not become immunodeficient (long-term non-progressors, LTNPs). $\mathrm{CD}^{+} \mathrm{T}$ cells are known to be important, but why are they more effective in some individuals than others? A recent paper in Nature Immunology showed that the increased expression of perforin by $\mathrm{CD}^{+} \mathrm{T}$ cells from LTNPs is important for their cytolytic activity (see the Highlight 'Quality control' in our November issue). But, since the first description of CD8 antiviral factor $(\mathrm{CAF})$ - which is secreted by stimulated $\mathrm{CD} 8^{+} \mathrm{T}$ cells from certain infected individuals - it has been recognized that soluble factors can inhibit virus replication also. Now, Zhang et al. report in Science the identification of human $\alpha$-defensins 1,2 and 3 as one of the main components of CAF.

Previous studies had indicated that $\beta$-chemokines (CCL3, CCL4 and CCL5) might account for the antiviral activity of CAF by competing with virus for binding to CCR5, which is used as a co-receptor for virus entry. However, this can only inhibit R5 viruses, and not $\mathrm{X} 4$ viruses, which use CXCR 4 as a co-receptor. Using protein-chip technology, Zhang et al. have shown that the $\alpha$-defensins carry out much of the anti-HIV activity of supernatants from stimulated $\mathrm{CD}^{+}$ $\mathrm{T}$ cells that is not attributable to $\beta$-chemokines.

The authors compared the protein mass spectra of supernatants from stimulated and unstimulated $\mathrm{CD} 8^{+}$ $\mathrm{T}$ cells of LTNPs, normal progressors and controls. Marked differences between stimulated and unstimulated spectra were observed - specifically, three peaks between 3.3 and $3.5 \mathrm{kD}$ that were present in stimulated cultures from LTNPs and some controls, but not progressors. The three peaks correspond to the molecular masses of human $\alpha$-defensins 1,2 and 3, and this result was confirmed by protein sequencing.

\title{
Untangling the TLRs
}

The Toll-like receptor (TLR) family consists of ten germline-encoded microbe sensors that are crucial for host defence. The theory is that each receptor triggers an innate immune response that is appropriate for the class of pathogen that the receptor recognizes. But, differences between TLR signal-transduction pathways that might result in such tailored responses have been hard to find. Now, two studies published in Nature show that the adaptor molecule TIRAP (also known as MAL) has a restricted role in a shared TLR2 and TLR4 signal-transduction pathway.

TIRAP is structurally similar to the adaptor protein MYD88, which links the TLRs and interleukin-1 receptors (IL-1Rs) to downstream signalling pathways. MYD88 is essential for the induction of cytokine secretion by all TLR ligands, but the lipopolysaccharide (LPS) receptor TLR4 can stimulate the upregulation of expression of co-stimulatory receptors on dendritic cells (DCs) in the absence of MYD88. Initial in vitro studies indicated that TIRAP might function in this MYD88-independent pathway. But, the Tirap-knockout mice described by Yamamoto $e t$ al. and Horng et al. show that this is not the case.

Horng and co-workers found that in response to triggering of TLR2 or TLR4, Tirap ${ }^{-/} \mathrm{B}$ cells had impaired proliferative responses and Tira $p^{-/}$DCs produced markedly reduced levels of proinflammatory cytokines. But, responses to a TLR9 ligand (CpG DNA) were normal in these cells, and injection of the TLR5 ligand flagellin into Tirap ${ }^{-1-}$ mice induced the expression of normal levels of cytokines. Yamamoto et al. showed that the production of pro-inflammatory cytokines in response to LPS and various ligands for TLR2 is impaired markedly in Tirap ${ }^{-/-}$ macrophages. Furthermore, Tira $p^{-/}$mice were completely resistant to LPS-induced shock. But, the responses of Tira $p^{-1-}$ macrophages to synthetic ligands for TLR7 and TLR3, and CPG DNA, were intact.

In contrast to $M y d 88^{-/-}$mice, both groups showed that IL-1 signalling is not compromised in Tirap ${ }^{-1-}$ mice. So, together, these papers show that TIRAP is dispensable for TLR3, TLR5, TLR7, TLR9 and IL-1R function, but has a specific role in TLR2 and TLR4 signal-transduction pathways.

Similar to MYD88, TIRAP has a crucial role in the activation of nuclear factor- $\mathrm{KB}$ and mitogen-activated protein kinases by TLR2 and TLR4. And Tirap ${ }^{-/}$mice and $M y d 88^{-1-}$ mice have similarly defective responses to LPS, which indicates that TIRAP might be involved in a MYD88dependent pathway. To test this, Yamamoto and co-workers generated $\mathrm{Myd} 88^{-1-}$ Tirap $^{-1-}$ mice. The LPS-induced upregulation of expression of co-stimulatory molecules on DCs (a MYD88-independent event) occurred normally in these doubleknockout mice, so TIRAP is clearly not part of the MYD88-independent pathway.

Jennifer Bell

(4) References and links

ORIGINAL RESEARCH PAPERS Yamamoto, M. et al. Essential role for TIRAP in activation of the signalling cascade shared by TLR2 and TLR4. Nature 420, 324-329 (2002) | Horng, T. et al. The adaptor molecule TIRAP provides signalling specificity for Toll-like receptors. Nature 420, 329-333 (2002)

FURTHER READING Medzhitov, R. Toll-like receptors and innate immunity. Nature Rev. Immunol. 1, 135-145 (2001) WEB SITE

Ruslan Medzhitov's lab:

http://info.med.yale.edu/immuno/fac_medzhitov.html 
Depletion of these molecules from culture supernatants eliminated activity against $\mathrm{X} 4$ viruses and markedly reduced activity against R5 viruses. Residual activity against R5 viruses could be neutralized by the addition of antibodies specific for the $\beta$-chemokines. Furthermore, synthetic and purified $\alpha$-defensins were shown to reduce HIV-1 replication in vitro.

So, we are one step closer to understanding the mystery of LTNPs, which can only aid the development of new therapeutics. It remains to be determined how the $\alpha$-defensins mediate their antiretroviral effects, and whether they are involved directly in non-progression.

KirstyMinton

(2) References and links ORIGINAL RESEARCH PAPER Zhang, L. et al. Contribution of human $\alpha$-defensin 1,2 and 3 to the anti-HIV-1 activity of CD8 antiviral factor. Science 298, 995-999 (2002)

\section{WEB SITE}

David Ho's lab:

http://www.rockefeller.edu/labheads/ho/ho.html

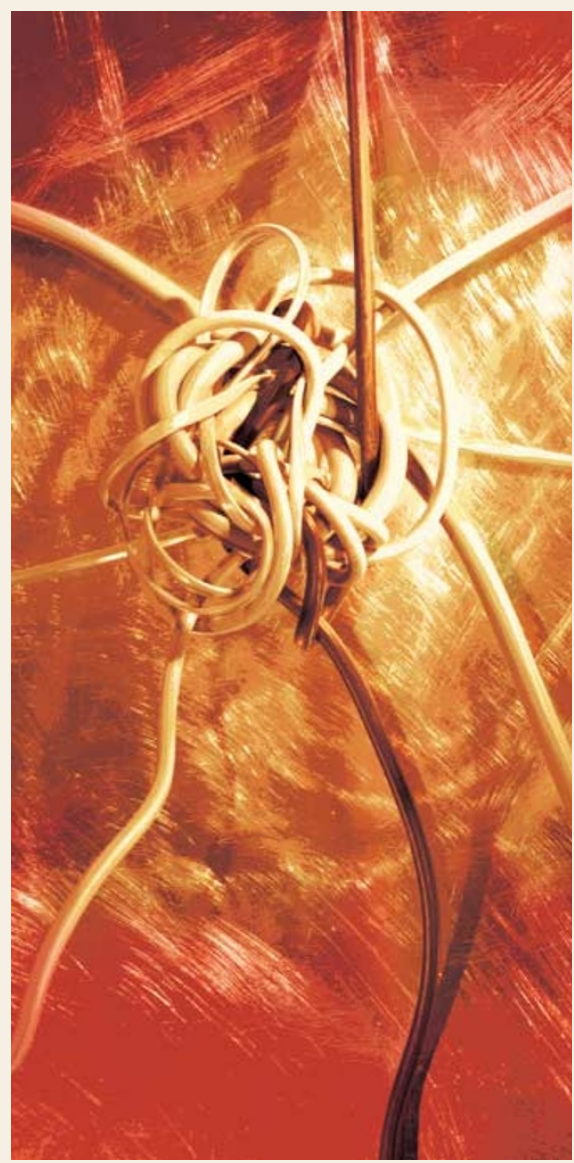

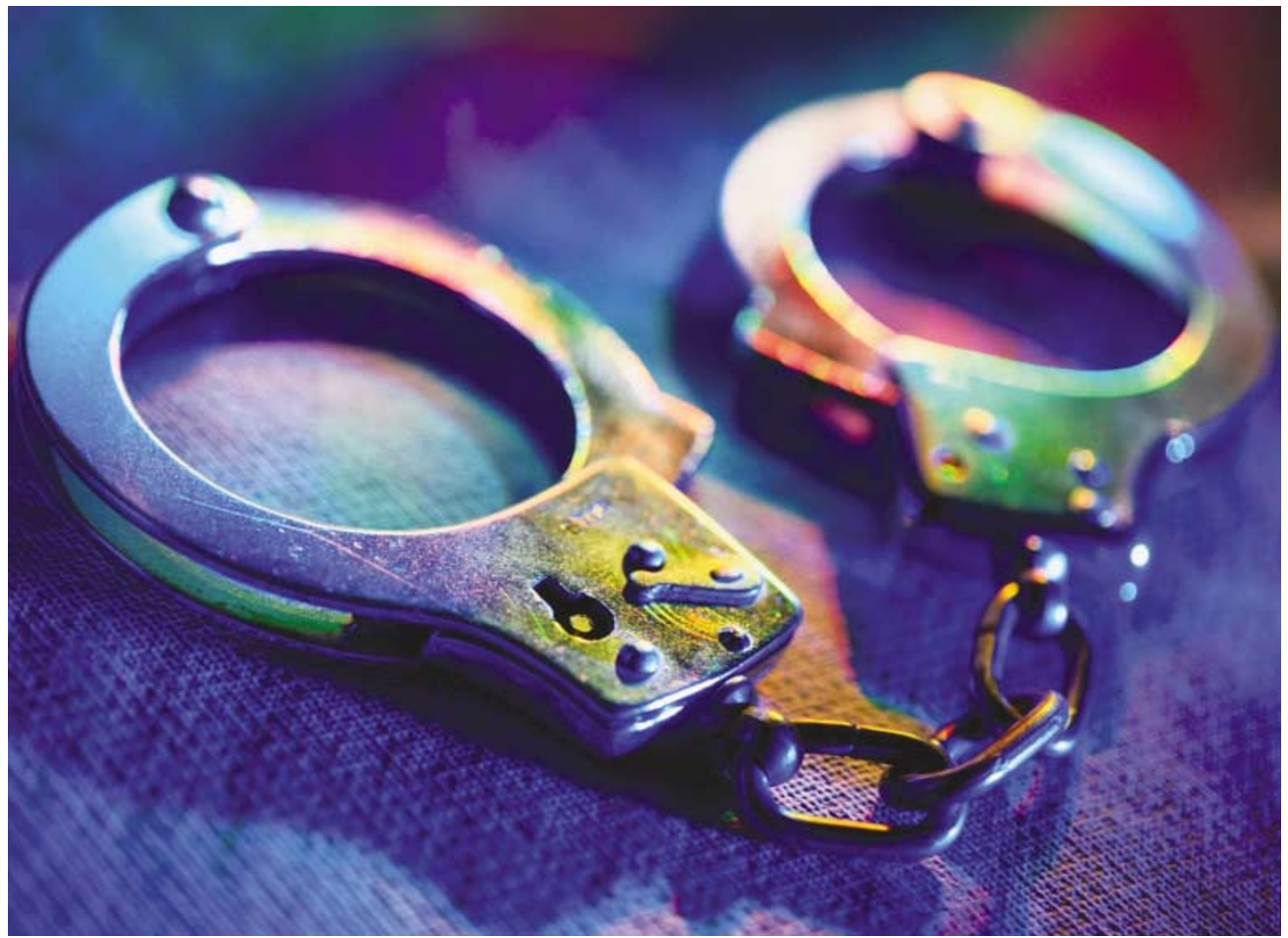

\section{TOLERANCE}

\section{DCs on the beat}

Dendritic cells (DCs) have long been recognized as the body's sentinels, but evidence is emerging that they are responsible also for policing the immune system. It has been proposed that DCs are 'on the beat' constantly, ingesting tissueassociated antigens and presenting them in local lymph nodes. In the absence of inflammation, this should result in the elimination of potentially harmful self-reactive $\mathrm{T}$ cells. Three papers in a recent issue of The Journal of Experimental Medicine provide further support for this model and indicate that $\mathrm{CD} 8 \alpha^{+}$DCs might be specialized for this task.

A previous study showed that the presentation of tissue-associated self-antigens by bonemarrow-derived cells in draining lymph nodes results in the deletion of self-reactive T cells. DCs have been implicated in this 'cross-tolerance', but the DC subpopulation(s) that is involved has not been pinpointed. Belz and colleagues generated mice that express a fusion protein containing two MHC class-I-restricted epitopes - an ovalbumin peptide and a herpes simplex virus (HSV) peptide - under the control of the rat insulin promoter. Ovalbumin-specific or HSV-specific TCRtransgenic $\mathrm{CD}^{+} \mathrm{T}$ cells that were injected into these mice were deleted rapidly. By sorting DC populations in the pancreatic lymph nodes, the authors showed that only CD8 $\alpha^{+}$DCs could stimulate a highly sensitive HSV-specific T-cell hybridoma, indicating that this DC population, which is implicated in cross-presentation, also mediates cross-tolerance.

The second paper addresses the issue of how tolerogenic DCs might acquire antigen in the steady state. An earlier study showed that CD8 $\alpha^{+}$ DCs are highly efficient at taking up and presenting antigens that are associated with dying cells; but, can this lead to tolerance? Liu et al. injected mice with transporter for antigen presentation (TAP)-deficient splenocytes that had been loaded with small amounts of ovalbumin protein and osmotically shocked to induce cell death. Because they lack TAP proteins, these cells cannot present their own antigens. The ovalbumin was processed and presented by CD8 $\alpha^{+}$DCs in the spleen. Transferred ovalbumin-specific TCRtransgenic $\mathrm{CD}^{+} \mathrm{T}$ cells proliferated initially, but were deleted subsequently, and the animals were rendered tolerant to ovalbumin. However, the maturation of DCs induced by a CD40-specific antibody resulted in immunity rather than tolerance. Although these data indicate that dying cells target $\mathrm{CD} 8 \alpha^{+} \mathrm{DCs}$, it is not known whether the cells that are targeted normally for presentation of self-antigens are living or dead.

The idea that the same $\mathrm{CD} 8 \alpha^{+} \mathrm{DC}$ subpopulation induces both cross-tolerance and cross-presentation is attractive. However, there might be dedicated immunogenic and tolerogenic DC subsets in the $C D 8 \alpha^{+}$DC population.

Moreover, studies by other groups have implicated other DC subsets in the induction of tolerance. A paper by Scheinecker and colleagues in the same issue shows that DCs presenting MHC class-II-restricted tissue-specific antigens in the gastric lymph nodes can be $\mathrm{CD}^{+}$or $\mathrm{CD}^{-}$, and $\mathrm{CD} 11 \mathrm{~b}^{+}$or $\mathrm{CD} 11 \mathrm{~b}^{-}$. Further studies are required to clarify these issues.

Jennifer Bell

6) References and links

ORIGiNAL RESEARCH PAPERS Belz, G. T. et al. The CD $8 \alpha^{+}$ dendritic cell is responsible for inducing peripheral self-tolerance to tissue-associated antigens. J. Exp. Med. 196, 1099-1104 (2002) | $\mathrm{Liu}, \mathrm{K}$. et al. Immune tolerance after delivery of dying cells to dendritic cells in situ. J. Exp. Med. 196, 1091-1097 (2002) | Scheinecker, C. et al. Constitutive presentation of a natural tissue autoantigen exclusively by dendritic cells in the draining lymph node. J. Exp. Med. 196, 1079-1090 (2002)

FURTHER READING Mougneau, E., Hugues, S. \& Glaichenhaus, N. Antigen presentation by dendritic cells in vivo. J. Exp. Med. 196 1013-1016 (2002) 